\title{
BMJ Open Protocol for a systematic review of the financial burden experienced by people affected by head and neck cancer
}

Nikki McCaffrey (D) , ${ }^{1,2}$ Lidia Engel $^{1}$

To cite: McCaffrey N, Engel L. Protocol for a systematic review of the financial burden experienced by people affected by head and neck cancer. BMJ Open 2022;12:e055213. doi:10.1136/ bmjopen-2021-055213

- Prepublication history and additional supplemental material for this paper are available online. To view these files, please visit the journal online (http://dx.doi.org/10.1136/ bmjopen-2021-055213).

Received 06 July 2021 Accepted 21 January 2022

Check for updates

(c) Author(s) (or their employer(s)) 2022. Re-use permitted under CC BY-NC. No commercial re-use. See rights and permissions. Published by BMJ.

${ }^{1}$ Deakin Health Economics, School of Health and Social Development, Deakin University, Burwood, Victoria, Australia

${ }^{2}$ Cancer Council Victoria, Melbourne, Victoria, Australia

Correspondence to Dr Nikki McCaffrey; nikki.mccaffrey@deakin.edu.au

\section{ABSTRACT}

Introduction Head and neck cancer (HNC) is the seventh most common cancer worldwide. Treatment may be associated with the inability to work and substantial out-of-pocket expenses. However, to date, there is little research synthesising quantitative evidence on the financial burden experienced by people affected by HNCs, including family members and informal carers. The purpose of this systematic review is to estimate out-ofpocket costs, reduced or lost income and informal care costs associated with HNC, identify categories of financial burden and investigate which costs predominate when considering financial burden in this population.

Methods and analysis A comprehensive search of peerreviewed literature will be conducted for articles published from 01/01/2010 to 19/03/2021 (CINAHL, Cochrane library, EconLit, Embase, Medline Complete). Published, Englishlanguage articles describing primary and secondary research directly related to the topic and quantitative cost data will be included. One researcher will complete the searches and screen results for potentially eligible studies. Three other researchers will independently screen the titles and abstracts of a subset of $30 \%$ citations, that is, $10 \%$ each. Full text articles will be independently screened by three reviewers. Any disagreement will be resolved by consensus among the team. Study and patient characteristics, cost categories and financial burden will be independently extracted by one reviewer and checked by a second. Methodological quality will be evaluated independently by two reviewers. Descriptive analyses will be undertaken and a narrative summary of the included studies will be provided.

Ethics and dissemination Ethics approval is not required to conduct this research because this is a planned systematic review of published literature. Findings will be presented at leading cancer and health economic conferences, published in a peer-reviewed journal and disseminated via website postings and social media channels.

Systematic review registration number CRD42021252929.

\section{INTRODUCTION}

Head and neck cancer (HNG) is ranked as one of the top 10 cancers globally with over 850000 new cases diagnosed each year. ${ }^{12}$ Incidence is anticipated to continue rising with more than one million new cases

\section{Strengths and limitations of this study}

The planned systematic review will provide insights into the financial burden faced by patients and families to inform the development of appropriate strategies for improving financial well-being in people affected by head and neck cancer.

- The systematic review protocol is reported according to the recommendations of the Preferred Reporting Items for Systematic Review and MetaAnalysis Protocols (PRISMA-P) statement and is registered with the International Prospective Register of Systematic Reviews (PROSPER0).

- The total number of studies investigating the financial burden experienced by people affected by head and neck cancer may be small; study methods may be heterogeneous; and cost estimates may be derived from diverse health financing systems, limiting study findings.

each year predicted by $2030 .{ }^{2}$ Generally, HNC begins in the squamous cells of the mouth, throat and nose and includes cancers of the oral cavity, nasal cavity and paranasal sinuses, salivary glands, pharynx and larynx, ${ }^{3}$ and thus far, there is no effective screening strategy for HNC. Meticulous physical examination is the key approach for early detection. ${ }^{4}$ Treatment may involve chemotherapy, radiotherapy, surgery or typically, a combination of these approaches, particularly in later stages. ${ }^{3}$ Many patients experience substantial functional impairment due to the location of the tumour, such as loss of speech, difficulties with swallowing and speech and challenges with eating ${ }^{5}$ and symptoms associated with HNC, and its treatment include pain, breathlessness, dry mouth, fatigue and depression. ${ }^{67}$

People affected by cancer can experience substantial out-of-pocket expenses associated with treatment costs, travel expenses and reduced household income from a diminished ability to continue paid employment. ${ }^{89}$ Informal carers, that is, people who provide ongoing, regular assistance to a person with chronic, progressive or life-limiting 
illness, typically without receiving payment for the assistance they provide, ${ }^{10-12}$ also report significant financial burden particularly related to reduced income and the costs of care time. ${ }^{13}{ }^{14}$ Financial burden has been associated with greater mortality risk, poorer quality of life, worse symptoms and reduced concordance with treatment advice. $^{15-18}$ Consequently, promoting financial well-being is a vital component of supportive care for people affected by cancer. Increasingly, clinical practice guidelines recommend discussion of the possible costs of cancer care and potential financial burden to patients and families, particularly in vulnerable groups such as low socioeconomic status populations. ${ }^{19}$ As socioeconomically deprived groups are disproportionally affected by $\mathrm{HNC},{ }^{20-22}$ managing financial burden is particular germane for people with this type of cancer.

While attempts have been made to summarise and quantify the financial burden experienced by people affected by a broad range of cancer types, ${ }^{83-27}$ thus far, such information for people impacted by HNC is lacking. This information will help inform the development of appropriate policies, programmes and strategies for improving financial well-being in this population. Consequently, the aim of this systematic review is to address this gap in knowledge by synthesising evidence on the constituents and magnitude of financial burden faced by patients and families affected by HNC.

\section{Review questions}

This systematic review addresses three related research questions:

1. What is the financial burden faced by people affected by HNC?

2. What are the main categories of out-of-pocket expenses faced by people affected by HNC?

3 . Which types of cost cause the greatest financial burden for people affected by HNC?

\section{METHODS}

This protocol is reported according to the recommendations of the Preferred Reporting Items for Systematic Review and Meta-Analysis Protocols (PRISMA-P) statement. $^{28}$

\section{Eligibility criteria}

Published, peer-reviewed, English-language articles reporting primary and secondary research directly related to the topic and quantitative cost data will be eligible for inclusion in the systematic review.

Inclusion criteria

- Studies including people affected by HNC, that is, patients, families and informal carers;

- Studies reporting and quantifying costs borne by patients and families; and

- Primary and secondary research directly related to the topic, that is, there will be no restrictions regarding research design.

Exclusion criteria
- Studies reporting qualitative data only;

- Studies which do not report cost estimates;

- Economic evaluations; and

- Non-English articles and those not peer reviewed, including comment or discussion papers, editorials, conference abstracts or papers and the grey literature.

\section{Search strategy}

A comprehensive search of the literature will be conducted from 1 January 2010 to the date of the search (CINAHL, Cochrane library, EconLit, Embase, MEDLINE Complete) to identify relevant published studies fitting the inclusion criteria. Studies published before 2010 were excluded due to more recent advances in HNC management which will affect the relative level of financial burden experienced by people with $\mathrm{HNC}^{29-31}$ Keywords and subject headings, derived from previously conducted systematic reviews of financial burden or toxicity in people affected by cancer ${ }^{92326}$ and the advice of the Deakin University School of Health and Social Development liaison librarian, include the following: patient, family, carer, caregivers, cancer survivors, HNC, head and neck neoplasms, financial burden, financial toxicity, financial stress, economic hardship, costs and cost analysis and economics. The search strategies are described in the online supplemental file.

Backwards and forwards citation tracing of included articles will be conducted for additional literature unidentified by the search. Searches will be restricted to the English language only. Search results will be downloaded into Thomson Reuters EndNote V. X9.2 (2019) libraries and Rayyan software ${ }^{32}$ will be used to manage records throughout the review.

\section{Selection process}

Step 1: The titles and abstracts of the studies identified by the search will be screened independently by one researcher (SK) to determine eligibility for inclusion in the review. Full text articles will be retrieved when there is insufficient information to judge relevance. Corresponding authors will be contacted to ascertain whether studies meet inclusion criteria if necessary. Three researchers (NM, LE, SA) will independently screen a subset of $30 \%$ citations, that is, $10 \%$ each. Agreement between the reviewers will be assessed using the Kappa statistic to determine if further duplicate title and abstract reviewing is required (Kappa $<0.4$, ie, fair agreement) ${ }^{33}$ If required, an additional $25 \%$ of citations will be screened (NM, LE, SA).

Step 2: The full text articles of potentially eligible studies will be retrieved and independently assessed by three reviewers (NM, LE, SK). Backwards and forwards citation tracing of included articles will be conducted for additional literature unidentified by the search.

Any disagreement will be resolved by consensus among the team.

\section{Risk of bias assessment}

The methodological quality of the included studies will be evaluated independently by two reviewers (NM, LE) 
using Larg and Moss's guide to critical evaluation of costof-illness studies ${ }^{34}$ and the Strengthening the Reporting of Observational Studies in Epidemiology guidelines for observational studies ${ }^{35}$ critical appraisal checklists. Any disagreement will be resolved by consensus among the team.

\section{Data collection}

A table will be created in Microsoft Office Excel 2013 to extract data from the included studies and summarise information about the constituents and magnitude of the financial burden for people affected by HNC. This software will be used to handle data throughout the review. The form will be piloted with two of the included articles and updated accordingly. Extracted information will include study characteristics (first author's name, publication date, country of origin, design, intervention), study population (age, gender, primary tumour site, sample size, subgroup), timeframe, costs categorised in line with the recommendations of the second panel on costeffectiveness in health and medicine (formal healthcare sector, informal healthcare sector and non-health care sectors), ${ }^{36}$ and lost patient income and lost informal carer income. The views of a third reviewer (SA) will be sought where there is ambiguity or disagreement.

\section{Data synthesis}

A narrative synthesis of the included studies will be provided in accordance with guidance from the Cochrane Collaboration. ${ }^{37}$ A meta-analysis will not be conducted given the anticipated heterogeneity between studies. ${ }^{38}$ Three tables summarising the key features of the included studies, cost components and quality assessment will be presented. Similarities and differences between the studies and results will be reported and cost estimates will be converted into 2020 Australian dollars using the CCEMG-EPPI-Centre Cost Converter. ${ }^{39}$ If feasible, average costs per category will be calculated and all costs will be converted to annual figures to enable comparability. All relevant studies will be included in the review and an assessment of how risk of bias may have affected the main results and outcome measures will be presented. Finally, the strength of the body of evidence will be independently assessed by two reviewers (NM, LE) using the GRADE guidelines. ${ }^{40}$ The views of a third reviewer (SA) will be sought where there is ambiguity or disagreement.

\section{ETHICS AND DISSEMINATION}

Ethics approval to conduct this research is not required because this study is a planned systematic review of published literature. The protocol is registered with PROSPERO (CRD42021252929). Findings will be presented at leading cancer and health economic conferences, published in a peer-reviewed journal and disseminated via website postings such as the Deakin University Institute for Health Transformation LinkedIn website and social media channels such as Twitter (eg, @DHE_ Deakin, @IHT_Deakin) and Facebook.
Acknowledgements Many thanks to the Deakin University School of Health and Social Development liaison librarian, Rachel West, for her advice on the search strategy, and Snehal Kulkarni (SK) and Siti Mohamad Asfia (SA) for agreeing to screen the titles and abstracts.

Contributors NM led the conception and design of the work with input from LE. NM drafted the work and LE revised the protocol and manuscript critically for comment. Both authors approved the final version to be published and are accountable for all aspects of the work. NM is the guarantor for the overall content.

Funding The authors have not declared a specific grant for this research from any funding agency in the public, commercial or not-for-profit sectors.

Competing interests None declared.

Patient consent for publication Not applicable.

Provenance and peer review Not commissioned; externally peer reviewed.

Supplemental material This content has been supplied by the author(s). It has not been vetted by BMJ Publishing Group Limited (BMJ) and may not have been peer-reviewed. Any opinions or recommendations discussed are solely those of the author(s) and are not endorsed by BMJ. BMJ disclaims all liability and responsibility arising from any reliance placed on the content. Where the content includes any translated material, BMJ does not warrant the accuracy and reliability of the translations (including but not limited to local regulations, clinical guidelines, terminology, drug names and drug dosages), and is not responsible for any error and/or omissions arising from translation and adaptation or otherwise.

Open access This is an open access article distributed in accordance with the Creative Commons Attribution Non Commercial (CC BY-NC 4.0) license, which permits others to distribute, remix, adapt, build upon this work non-commercially, and license their derivative works on different terms, provided the original work is properly cited, appropriate credit is given, any changes made indicated, and the use is non-commercial. See: http://creativecommons.org/licenses/by-nc/4.0/.

ORCID iD

Nikki McCaffrey http://orcid.org/0000-0003-3684-3723

\section{REFERENCES}

1 GBD 2016 Disease and Injury Incidence and Prevalence Collaborators. Global, regional, and national incidence, prevalence, and years lived with disability for 328 diseases and injuries for 195 countries, 1990-2016: a systematic analysis for the global burden of disease study 2016. Lancet 2017;390:1211-59.

2 Bray F, Ferlay J, Soerjomataram I, et al. Global cancer statistics 2018: GLOBOCAN estimates of incidence and mortality worldwide for 36 cancers in 185 countries. CA Cancer J Clin 2018;68:394-424.

3 Chow LQM. Head and neck cancer. N Engl J Med 2020;382:60-72.

4 Johnson DE, Burtness B, Leemans CR, et al. Head and neck squamous cell carcinoma. Nat Rev Dis Primers 2020;6:92.

5 Longacre ML, Ridge JA, Burtness BA, et al. Psychological functioning of caregivers for head and neck cancer patients. Oral Oncol 2012;48:18-25.

6 Meregaglia M, Cairns J. A systematic literature review of health state utility values in head and neck cancer. Health Qual Life Outcomes 2017;15:174.

7 Pollaers K, Massingham I, Friedland PL, et al. The economic burden of oral squamous cell carcinoma in Australia. J Oral Pathol Med 2019;48:588-94.

8 Mols F, Tomalin B, Pearce A, et al. Financial toxicity and employment status in cancer survivors. A systematic literature review. Support Care Cancer 2020;28:5693-708.

9 Azzani M, Roslani AC, Su TT, . The perceived cancer-related financial hardship among patients and their families: a systematic review. Support Care Cancer 2015;23:889-98.

10 McCaffrey N, Bucholc J, Rand S, et al. Head-To-Head comparison of the psychometric properties of 3 Carer-Related Preference-Based instruments. Value Health 2020;23:1477-88.

11 McCaffrey N, Cassel JB, Coast J. Bringing the economic cost of informal caregiving into focus. Palliat Med 2015;29:866-7.

12 Al-Janabi H, McCaffrey N, Ratcliffe J. Carer preferences in economic evaluation and healthcare decision making. Patient 2013;6:235-9.

13 Coumoundouros C, Ould Brahim L, Lambert SD, et al. The direct and indirect financial costs of informal cancer care: a scoping review. Health Soc Care Community 2019;27:e622-36.

14 Engel L, Ajdukovic M, Bucholc J, et al. Valuation of informal care provided to people living with dementia: a systematic literature review. Value Health. In Press 2021;24:1863-70. 
15 Mady LJ, Lyu L, Owoc MS, et al. Understanding financial toxicity in head and neck cancer survivors. Oral Oncol 2019;95:187-93.

16 Arastu A, Patel A, Mohile SG, et al. Assessment of financial toxicity among older adults with advanced cancer. JAMA Netw Open 2020;3:e2025810-e10.

17 Lu L, O'Sullivan E, Sharp L. Cancer-Related financial hardship among head and neck cancer survivors: risk factors and associations with health-related quality of life. Psychooncology 2019;28:863-71.

18 Massa ST, Osazuwa-Peters N, Adjei Boakye E, et al. Comparison of the financial burden of survivors of head and neck cancer with other cancer survivors. JAMA Otolaryngol Head Neck Surg 2019;145:239-49.

19 Agarwal A, Livingstone A, Karikios D. Discussing the costs and financial burden of cancer and its treatment: a systematic review of clinical guidelines. Research Square, 2021.

20 Conway DI, Brenner DR, McMahon AD, et al. Estimating and explaining the effect of education and income on head and neck cancer risk: INHANCE Consortium pooled analysis of 31 casecontrol studies from 27 countries. Int J Cancer 2015;136:1125-39.

21 Hwang E, Johnson-Obaseki S, McDonald JT, et al. Incidence of head and neck cancer and socioeconomic status in Canada from 1992 to 2007. Oral Oncol 2013;49:1072-6.

22 Aupérin A. Epidemiology of head and neck cancers: an update. Curr Opin Oncol 2020;32:178-86.

23 Altice CK, Banegas MP, Tucker-Seeley RD, et al. Financial Hardships experienced by cancer survivors: a systematic review. J Nat/ Cancer Inst 2017;109. doi:10.1093/jnci/djw205. [Epub ahead of print: 2010 2016].

24 Longo CJ, Fitch MI, Banfield L, et al. Financial toxicity associated with a cancer diagnosis in publicly funded healthcare countries: a systematic review. Support Care Cancer 2020;28:4645-65.

25 Carrera PM, Kantarjian HM, Blinder VS. The financial burden and distress of patients with cancer: understanding and stepping-up action on the financial toxicity of cancer treatment. CA Cancer J Clin 2018;68:153-65.

26 Gordon LG, Merollini KMD, Lowe A, et al. A Systematic Review of Financial Toxicity Among Cancer Survivors: We Can't Pay the CoPay. Patient 2017;10:295-309.

27 Bygrave A, Whittaker K, Paul C, et al. Australian experiences of outof-pocket costs and financial burden following a cancer diagnosis: a systematic review. Int J Environ Res Public Health 2021;18:2422.
28 Moher D, Shamseer L, Clarke M, et al. Preferred reporting items for systematic review and meta-analysis protocols (PRISMA-P) 2015 statement. Syst Rev 2015;4:1-9.

29 Saleh K, Eid R, Haddad FG, et al. New developments in the management of head and neck cancer - impact of pembrolizumab. Ther Clin Risk Manag 2018;14:295-303.

30 Mendenhall WM, Dagan R, Bryant CM, et al. Radiation oncology for head and neck cancer: current standards and future changes. Oral Maxillofac Surg Clin North Am 2019;31:31-8.

31 Alsahafi E, Begg K, Amelio I, et al. Clinical update on head and neck cancer: molecular biology and ongoing challenges. Cell Death Dis 2019;10:540.

32 Ouzzani M, Hammady H, Fedorowicz Z, et al. Rayyan-a web and mobile APP for systematic reviews. Syst Rev 2016;5:210.

33 Orwin EG. Evaluating coding decisions. In: Cooper H, LV H, eds. The Handbook of research synthesis. New York: Russell Sage Foundation, 1994

34 Larg A, Moss JR. Cost-Of-Illness studies: a guide to critical evaluation. Pharmacoeconomics 2011;29:653-71.

35 von Elm E, Altman DG, Egger M, et al. The strengthening the reporting of observational studies in epidemiology (STROBE) statement: guidelines for reporting observational studies. J Clin Epidemiol 2008;61:344-9.

36 Sanders GD, Neumann PJ, Basu A, et al. Recommendations for conduct, methodological practices, and reporting of costeffectiveness analyses: second panel on cost-effectiveness in health and medicine. JAMA 2016;316:1093-103.

37 Ryan R, Cochrane Consumers and Communication Review Group. Cochrane consumers and communication review group: data synthesis and analysis, 2013.

38 Jacobsen E, Boyers D, Avenell A. Challenges of systematic reviews of economic evaluations: a review of recent reviews and an obesity case study. Pharmacoeconomics 2020;38:259-67.

39 Shemilt I, Thomas J, Morciano M. A web-based tool for adjusting costs to a specific target currency and price year. Evidence \& Policy: A Journal of Research, Debate and Practice 2010;6:51-9.

40 Andrews JC, Schünemann HJ, Oxman AD, et al. Grade guidelines: 15. going from evidence to recommendation-determinants of a recommendation's direction and strength. J Clin Epidemiol 2013;66:726-35 